\title{
The weak type inequality for the Walsh system
}

\author{
by \\ Ushangi Goginava (Tbilisi)
}

\begin{abstract}
The main aim of this paper is to prove that the maximal operator $\sigma^{\#}$ is bounded from the Hardy space $H_{1 / 2}$ to weak- $L_{1 / 2}$ and is not bounded from $H_{1 / 2}$ to $L_{1 / 2}$.
\end{abstract}

1. Introduction. The first result on a.e. convergence of the Walsh-Fejér means $\sigma_{n} f$ is due to Fine [1]. Later, Schipp [6] showed that the maximal operator $\sigma^{*} f$ is of weak type $(1,1)$, from which the a.e. convergence follows by standard arguments. Schipp's result implies by interpolation also the boundedness of $\sigma^{*}: L_{p} \rightarrow L_{p}(1<p \leq \infty)$. This fails to hold for $p=1$ but Fujii [2] proved that $\sigma^{*}$ is bounded from the dyadic Hardy space $H_{1}$ to $L_{1}$ (see also Simon [8]). Fujii's theorem was extended by Weisz [11], who proved that the maximal operator of the Fejér means of the one-dimensional Walsh-Fourier series is bounded from the martingale Hardy space $H_{p}(I)$ to $L_{p}(I)$ for $p>1 / 2$. Simon [9] gave an example to show that this does not hold for $0<p<1 / 2$. In the endpoint case $p=1 / 2$ Weisz [14] proved that $\sigma^{*}$ is bounded from the Hardy space $H_{1 / 2}(I)$ to weak- $L_{1 / 2}(I)$.

For the two-dimensional Walsh-Fourier series Weisz [12] proved that the maximal operator

$$
\sigma^{*} f=\sup _{n \geq 1} \frac{1}{n}\left|\sum_{j=1}^{n} S_{j, j}(f)\right|
$$

is bounded from the two-dimensional dyadic martingale Hardy space $H_{p}$ to $L_{p}$ for $p>2 / 3$, and Goginava [4] generalized this result to $d$-dimensional Walsh-Fourier series. The a.e. convergence of the arithmetic means of square partial sums of double Vilenkin-Fourier series was studied by Gát [3].

The main aim of this paper is to prove that the maximal operator of the Marcinkiewicz-Fejér means of the double Walsh-Fourier series is bounded from the dyadic Hardy space $H_{1 / 2}$ to weak- $L_{1 / 2}$ and is not bounded from

2000 Mathematics Subject Classification: Primary 42C10.

Key words and phrases: Walsh function, Hardy space, maximal operator.

This paper was written during the author's visit at the College of Nyíregyháza, Hungary. 
$H_{1 / 2}$ to $L_{1 / 2}$ provided that the supremum in the maximal operator is taken over spatial indices.

2. Definitions and ntation. Let $\mathbb{P}$ denote the set of positive integers, and $\mathbb{N}:=\mathbb{P} \cup\{0\}$. Denote by $Z_{2}$ the discrete cyclic group of order 2 , that is, $Z_{2}=\{0,1\}$, where the group operation is the modulo 2 addition and every subset is open. The Haar measure on $Z_{2}$ is such that the measure of a singleton is $1 / 2$. Let $G$ be the complete direct product of a countably infinite number of copies of the compact group $Z_{2}$. Elements of $G$ are of the form $x=\left(x_{0}, x_{1}, \ldots, x_{k}, \ldots\right)$ with $x_{k} \in\{0,1\}(k \in \mathbb{N})$. The group operation on $G$ is coordinatewise addition, and the measure (denoted by $\mu$ ) and the topology are the product measure and topology. The compact Abelian group $G$ is called the Walsh group. A base of neighborhoods of $x \in G$ can be given in the following way:

$$
\begin{aligned}
& I_{0}(x):=G, \\
& I_{n}(x):=I_{n}\left(x_{0}, \ldots, x_{n-1}\right):=\left\{y \in G: y=\left(x_{0}, \ldots, x_{n-1}, y_{n}, y_{n+1}, \ldots\right)\right\}
\end{aligned}
$$

for $n \in \mathbb{N}$. These sets are called the dyadic intervals. Let $0=(0,0, \ldots) \in G$ denote the null element of $G, I_{n}:=I_{n}(0)(n \in \mathbb{N}), \bar{I}_{n}:=G \backslash I_{n}$. Set $e_{n}:=(0, \ldots, 0,1,0, \ldots) \in G$ with the $n$th coordinate 1 , and the other zeros. Define

$$
x_{i, j}:=\sum_{s=i}^{j} x_{s} e_{s}, \quad x_{i, i-1}=0 .
$$

For $k \in \mathbb{N}$ and $x \in G$ set

$$
r_{k}(x):=(-1)^{x_{k}},
$$

the $k$ th Rademacher function. If $n \in \mathbb{N}$, then $n=\sum_{i=0}^{\infty} n_{i} 2^{i}$, where $n_{i} \in$ $\{0,1\}(i \in \mathbb{N})$, i.e. $n$ is expressed in the number system of base 2 . Define $|n|:=\max \left\{j \in \mathbb{N}: n_{j} \neq 0\right\}$, that is, $2^{|n|} \leq n<2^{|n|+1}$.

The Walsh-Paley system is defined as the sequence of Walsh-Paley functions

$$
w_{n}(x):=\prod_{k=0}^{\infty}\left(r_{k}(x)\right)^{n_{k}}=r_{|n|}(x)(-1)^{\sum_{k=0}^{|n|-1} n_{k} x_{k}} \quad(x \in G, n \in \mathbb{P}) .
$$

The Walsh-Dirichlet kernel is defined by

$$
D_{n}(x)=\sum_{k=0}^{n-1} w_{k}(x) \text {. }
$$

Recall that

$$
D_{2^{n}}(x)= \begin{cases}2^{n} & \text { if } x \in I_{n} \\ 0 & \text { if } x \in \bar{I}_{n}\end{cases}
$$


The rectangular partial sums of the 2-dimensional Walsh-Fourier series are defined as follows:

$$
S_{M, N} f\left(x^{1}, x^{2}\right):=\sum_{i=0}^{M-1} \sum_{j=0}^{N-1} \widehat{f}(i, j) w_{i}\left(x^{1}\right) w_{j}\left(x^{2}\right),
$$

where the number

$$
\widehat{f}(i, j)=\int_{G \times G} f\left(x^{1}, x^{2}\right) w_{i}\left(x^{1}\right) w_{j}\left(x^{2}\right) d \mu\left(x^{1}, x^{2}\right)
$$

is said to be the $(i, j)$ th Walsh-Fourier coefficient of the function $f$.

The norm (or quasinorm) of the space $L_{p}(G \times G)$ is defined by

$$
\|f\|_{p}:=\left(\int_{G \times G}\left|f\left(x^{1}, x^{2}\right)\right|^{p} d \mu\left(x^{1}, x^{2}\right)\right)^{1 / p} \quad(0<p<\infty) .
$$

The space weak- $L_{p}(G \times G)$ consists of all measurable functions $f$ for which

$$
\|f\|_{\text {weak }-L_{p}(G \times G)}:=\sup _{\lambda>0} \lambda \mu(|f|>\lambda)^{1 / p}<\infty .
$$

The $\sigma$-algebra generated by the dyadic 2-dimensional cube $I_{k}\left(x^{1}\right) \times I_{k}\left(x^{2}\right)$ of measure $2^{-k} \times 2^{-k}$ will be denoted by $F_{k}(k \in \mathbb{N})$.

Denote by $f=\left(f^{(n)}, n \in \mathbb{N}\right)$ a one-parameter martingale with respect to $\left(F_{n}, n \in \mathbb{N}\right.$ ) (for details, see e.g. $\left.[10,13]\right)$. The maximal function of the martingale $f$ is defined by

$$
f^{*}=\sup _{n \in \mathbb{N}}\left|f^{(n)}\right| .
$$

In case $f \in L_{1}(G \times G)$, the maximal function can also be given by

$$
\begin{aligned}
f^{*}\left(x^{1}, x^{2}\right)=\sup _{n \geq 1} \frac{1}{\mu\left(I_{n}\left(x^{1}\right) \times I_{n}\left(x^{2}\right)\right)}\left|\int_{I_{n}\left(x^{1}\right) \times I_{n}\left(x^{2}\right)} f\left(u^{1}, u^{2}\right) d \mu\left(u^{1}, u^{2}\right)\right|, \\
\quad\left(x^{1}, x^{2}\right) \in G \times G .
\end{aligned}
$$

For $0<p<\infty$ the Hardy martingale space $H_{p}(G \times G)$ consists of all martingales for which

$$
\|f\|_{H_{p}}:=\left\|f^{*}\right\|_{p}<\infty
$$

If $f \in L_{1}(G \times G)$ then it is easy to show that the sequence $\left(S_{2^{n}, 2^{n}}(f)\right.$ : $n \in \mathbb{N})$ is a martingale. If $f$ is a martingale, that is, $f=\left(f^{(0)}, f^{(1)}, \ldots\right)$, then the Walsh-Fourier coefficients must be defined in a somewhat different way:

$$
\widehat{f}(i, j)=\lim _{k \rightarrow \infty} \int_{G \times G} f^{(k)}\left(x^{1}, x^{2}\right) w_{i}\left(x^{1}\right) w_{j}\left(x^{2}\right) d \mu\left(x^{1}, x^{2}\right) .
$$

The Walsh-Fourier coefficients of $f \in L_{1}(G \times G)$ are the same as those of the martingale $\left(S_{2^{n}, 2^{n}}(f): n \in \mathbb{N}\right)$ obtained from $f$. 
For $n=1,2, \ldots$ and a martingale $f$ the Marcinkiewicz-Fejér means of order $2^{n}$ of the 2-dimensional Walsh-Fourier series of the function $f$ are given by

$$
\sigma_{2^{n}} f\left(x^{1}, x^{2}\right)=\frac{1}{2^{n}} \sum_{j=0}^{2^{n}-1} S_{j, j} f\left(x^{1}, x^{2}\right) .
$$

For the martingale $f$ we consider the maximal operator

$$
\sigma^{\#} f=\sup _{n}\left|\sigma_{2^{n}} f\left(x^{1}, x^{2}\right)\right| .
$$

The 2-dimensional Marcinkiewicz-Fejér kernel of order $2^{n}$ of the 2-dimensional Walsh-Fourier series is defined by

$$
K_{2^{n}}\left(x^{1}, x^{2}\right):=\frac{1}{2^{n}} \sum_{k=0}^{2^{n}-1} D_{k}\left(x^{1}\right) D_{k}\left(x^{2}\right) .
$$

It is easy to show that

$$
\sigma_{2^{n}} f\left(x^{1}, x^{2}\right)=\int_{G \times G} f\left(t^{1}, t^{2}\right) K_{2^{n}}\left(x^{1}+t^{1}, x^{2}+t^{2}\right) d \mu\left(t^{1}, t^{2}\right) .
$$

A bounded measurable function $a$ is a p-atom if there exists a dyadic 2-dimensional cube $I \times I$ such that

(a) $\int_{I \times I} a d \mu=0$;

(b) $\|a\|_{\infty} \leq \mu(I \times I)^{-1 / p}$;

(c) $\operatorname{supp} a \subset I \times I$.

\section{Formulation of main results}

THEOREM 1. The maximal operator $\sigma^{\#}$ is bounded from the Hardy space $H_{1 / 2}(G \times G)$ to weak- $L_{1 / 2}(G \times G)$.

THEOREM 2. The maximal operator $\sigma^{\#}$ is not bounded from $H_{1 / 2}(G \times G)$ to $L_{1 / 2}(G \times G)$.

Corollary 1. Let $p>1 / 2$. Then $\sigma^{\#}$ is bounded from the Hardy space $H_{p}(G \times G)$ to $L_{p}(G \times G)$.

CoRollary 2. Let $0<p<1 / 2$. Then $\sigma^{\#}$ is not bounded from $H_{p}(G \times G)$ to weak $-L_{p}(G \times G)$.

4. Auxiliary propositions. We shall need the following lemmas (see $[5,13])$.

Lemma 1 (Weisz). Suppose that an operator $V$ is sublinear and, for some $0<p<1$,

$$
\sup _{\varrho>0} \varrho^{p} \mu\{x \in(G \times G) \backslash(I \times I):|V a(x)|>\varrho\} \leq c_{p}<\infty
$$


for every p-atom a, where I denote the support of the atom. If $V$ is bounded from $L_{p_{1}}$ to $L_{p_{1}}$ for a fixed $1<p_{1} \leq \infty$, then

$$
\|V f\|_{\text {weak- } L_{p}(G \times G)} \leq c_{p}\|f\|_{H_{p}} .
$$

Lemma 2 (Nagy). Let $A, m, n \in \mathbb{N}, m \leq n<A$, and $\left(x^{1}, x^{2}\right) \in\left(I_{m} \backslash I_{m+1}\right)$ $\times\left(I_{n} \backslash I_{n+1}\right)$. Then

$$
K_{2^{A}}\left(x^{1}, x^{2}\right)
$$

$$
= \begin{cases}0 & \text { if } \exists i \in B_{1}, x_{i}^{1} \neq x_{i}^{2}, \\ 0 & \text { if } \forall i \in B_{1}, x_{i}^{1}=x_{i}^{2}, \exists s \in B_{2}, x^{1}-e_{m}-e_{s} \notin I_{n+1}, x_{s}^{1}=1, \\ 2^{s+m-2} & \text { if } \forall i \in B_{1}, x_{i}^{1}=x_{i}^{2}, \exists s \in B_{2}, x^{1}-e_{s}-e_{m} \in I_{n+1}, x_{s}^{1}=1, \\ 2^{2 m-1} & \text { if } x^{1}-e_{m} \in I_{n+1}, \forall i \in B_{1}, x_{i}^{1}=x_{i}^{2},\end{cases}
$$

where $B_{1}=\{n+1, \ldots, A-1\}, B_{2}=\{m+1, \ldots, n\}$.

Lemma 3 (Nagy). Let $A, s, l \in \mathbb{N},\left(x^{1}, x^{2}\right) \in I_{A} \times\left(I_{l} \backslash I_{l+1}\right)$ and $l<$ $s+l<A$. Then

$K_{2^{A}}\left(x^{1}, x^{2}\right)$

$$
= \begin{cases}0 & \text { if } \exists s, l<s+l<A, x^{2}-x_{l}^{2} e_{l}-e_{s+l} \notin I_{A}, x_{s+l}^{2} \neq 0, \\ 2^{2 l+s-2} & \text { if } \exists s, l<s+l<A, x^{2}-x_{l}^{2} e_{l}-e_{s+l} \in I_{A}, x_{s+l}^{2} \neq 0, \\ 2^{l-2} n(A, l) & \text { if } x^{2}-x_{l}^{2} e_{l} \in I_{A},\end{cases}
$$

where $n(A, l)=\left[-2^{l-A}\left(2^{A}-2^{l-1}+1 / 2\right)-\left(2^{A}-2\right)\right]$.

Lemma $4([4])$. Let $\left(x^{1}, x^{2}\right) \in \bar{I}_{N} \times \bar{I}_{N}$. Then

$$
\begin{aligned}
& \int_{I_{N} \times I_{N}} K_{2^{A}}\left(x^{1}+t^{1}, x^{2}+t^{2}\right) d \mu\left(t^{1}, t^{2}\right) \\
& \leq \frac{c}{2^{3 N}}\left|\sum_{j=1}^{2^{N}-1} D_{j}\left(x^{1}\right) D_{j}\left(x^{2}\right)\right|, \quad A \geq N .
\end{aligned}
$$

Lemma $5([4])$. Let $\left(x^{1}, x^{2}\right) \in I_{N} \times \bar{I}_{N}$. Then

$$
\begin{aligned}
& \int_{I_{N} \times I_{N}} K_{2^{A}}\left(x^{1}+t^{1}, x^{2}+t^{2}\right) d \mu\left(t^{1}, t^{2}\right) \\
& \quad \leq \frac{c}{2^{3 N}}\left\{\left|\sum_{j=1}^{2^{N}-1} D_{j}\left(x^{1}\right) D_{j}\left(x^{2}\right)\right|+2^{N}\left|\sum_{j=1}^{2^{N}-1} D_{j}\left(x^{2}\right)\right|\right\}, \quad A \geq N .
\end{aligned}
$$


Lemma 6. Let $\left(x^{1}, x^{2}\right) \in\left(I_{m} \backslash I_{m+1}\right) \times\left(I_{n} \backslash I_{n+1}\right), n \geq m, m, n=$ $0, \ldots, N-1, A>N$. Then

$$
\begin{aligned}
& \int_{I_{N} \times I_{N}} K_{2^{A}}\left(x^{1}+t^{1}, x^{2}+t^{2}\right) d \mu\left(t^{1}, t^{2}\right) \\
& \quad \leq \frac{c 2^{m-n}}{2^{3 N}} \sum_{r=m+1}^{n+1} 2^{r} D_{2^{n+1}}\left(x^{1}+e_{m}+e_{r}\right) D_{2^{N}}\left(x^{2}+e_{n}+x_{n+1, N-1}^{1}\right) .
\end{aligned}
$$

Proof. From Lemma 2 and by (1) we can write the following estimate:

$$
\begin{aligned}
& \left|\sum_{j=1}^{2^{N}-1} D_{j}\left(x^{1}\right) D_{j}\left(x^{2}\right)\right| \\
& \quad \leq c 2^{m-n} \sum_{r=m+1}^{n+1} 2^{r} D_{2^{n+1}}\left(x^{1}+e_{m}+e_{r}\right) D_{2^{N}}\left(x^{2}+e_{n}+x_{n+1, N-1}^{1}\right) .
\end{aligned}
$$

Applying Lemma 4 we complete the proof.

Lemma 7. Let $\left(x^{1}, x^{2}\right) \in I_{N} \times\left(I_{l} \backslash I_{l+1}\right), l=0, \ldots, N-1, A>N$. Then

$$
\int_{I_{N} \times I_{N}} K_{2^{A}}\left(x^{1}+t^{1}, x^{2}+t^{2}\right) d \mu\left(t^{1}, t^{2}\right) \leq \frac{c 2^{l}}{2^{3 N}} \sum_{m=l+1}^{N} 2^{m} D_{2^{N}}\left(x^{2}+e_{l}+e_{m}\right) \text {. }
$$

Proof. Since (see [7] and (1))

$$
\left|\sum_{j=1}^{2^{N}-1} D_{j}\left(x^{2}\right)\right| \leq c \sum_{j=0}^{N} 2^{j} D_{2^{N}}\left(x^{2}+e_{j}\right)=c 2^{l} D_{2^{N}}\left(x^{2}+e_{l}\right)
$$

and (see Lemma 3)

$$
\left|\sum_{j=1}^{2^{N}-1} D_{j}\left(x^{1}\right) D_{j}\left(x^{2}\right)\right| \leq c 2^{l} \sum_{m=l+1}^{N-1} 2^{m} D_{2^{N}}\left(x^{2}+e_{m}\right),
$$

from Lemma 4 we obtain

$$
\begin{aligned}
\int_{I_{N} \times I_{N}} K_{2^{A}} & \left(x^{1}+t^{1}, x^{2}+t^{2}\right) d \mu\left(t^{1}, t^{2}\right) \\
& \leq \frac{c 2^{l}}{2^{3 N}}\left\{\sum_{m=l+1}^{N-1} 2^{m} D_{2^{N}}\left(x^{2}+e_{l}+e_{m}\right)+2^{N} D_{2^{N}}\left(x^{2}+e_{l}\right)\right\} \\
& \leq \frac{c 2^{l}}{2^{3 N}} \sum_{m=l+1}^{N} 2^{m} D_{2^{N}}\left(x^{2}+e_{l}+e_{m}\right) .
\end{aligned}
$$




\section{Proofs of main results}

Proof of Theorem 1. We shall apply Lemma 1; we may suppose that $a \in L_{\infty}$ is a $1 / 2$-atom with support $I_{N} \times I_{N}$. Since $\sigma_{2^{A}} a\left(x^{1}, x^{2}\right)=0$ for $A \leq N$, we may assume that $A>N$.

Suppose that $\varrho=c 2^{\lambda}$ for some $\lambda \in \mathbb{N}$.

It is evident that

$$
\begin{aligned}
& \mu\left\{\left(x^{1}, x^{2}\right) \in \overline{I_{N} \times I_{N}}:\left|\sigma^{\#} a\left(x^{1}, x^{2}\right)\right|>c 2^{\lambda}\right\} \\
&= \mu\left\{\left(x^{1}, x^{2}\right) \in \bar{I}_{N} \times \bar{I}_{N}:\left|\sigma^{\#} a\left(x^{1}, x^{2}\right)\right|>c 2^{\lambda}\right\} \\
&+\mu\left\{\left(x^{1}, x^{2}\right) \in I_{N} \times \bar{I}_{N}:\left|\sigma^{\#} a\left(x^{1}, x^{2}\right)\right|>c 2^{\lambda}\right\} \\
&+\mu\left\{\left(x^{1}, x^{2}\right) \in \bar{I}_{N} \times I_{N}:\left|\sigma^{\#} a\left(x^{1}, x^{2}\right)\right|>c 2^{\lambda}\right\} .
\end{aligned}
$$

Let $\left(x^{1}, x^{2}\right) \in\left(I_{m} \backslash I_{m+1}\right) \times\left(I_{n} \backslash I_{n+1}\right), 0 \leq m \leq n<N$. Then from Lemma 6 we have

$$
\begin{aligned}
& \sigma^{\#} a\left(x^{1}, x^{2}\right) \\
& \leq c\|a\|_{\infty} \sup _{A \geq N} \int_{I_{N} \times I_{N}} K_{2^{A}}\left(x^{1}+t^{1}, x^{2}+t^{2}\right) d \mu\left(t^{1}, t^{2}\right) \\
& \leq \frac{c 2^{4 N} 2^{m-n}}{2^{3 N}} \sum_{r=m+1}^{n+1} 2^{r} D_{2^{n+1}}\left(x^{1}+e_{m}+e_{r}\right) D_{2^{N}}\left(x^{2}+e_{n}+x_{n+1, N-1}^{1}\right) \\
& =c 2^{N+m-n} \sum_{r=m+1}^{n+1} 2^{r} D_{2^{n+1}}\left(x^{1}+e_{m}+e_{r}\right) D_{2^{N}}\left(x^{2}+e_{n}+x_{n+1, N-1}^{1}\right) .
\end{aligned}
$$

Define

$$
\begin{aligned}
\sigma_{1}^{\#}\left(x^{1}, x^{2}\right):= & c 2^{N+m-n} \\
& \times \sum_{r=m+1}^{n+1} 2^{r} D_{2^{n+1}}\left(x^{1}+e_{m}+e_{r}\right) D_{2^{N}}\left(x^{2}+e_{n}+x_{n+1, N-1}^{1}\right) .
\end{aligned}
$$

It is evident (see (1)) that $\sigma_{1}^{\#}\left(x^{1}, x^{2}\right) \neq 0$ implies that

$$
x^{1} \in I_{N}\left(\overline{0}, x_{m}^{1}=1, \overline{0}, x_{l}^{1}=1, \overline{0}, x_{n+1}^{1}, \ldots, x_{N-1}^{1}\right)
$$

and

$$
x^{2} \in I_{N}\left(\overline{0}, x_{n}^{2}=1, x_{n+1}^{1}, \ldots, x_{N-1}^{1}\right)
$$

for some $l$ with $m<l \leq n+1$, where $\overline{0}$ denotes a string of zeros. Consequently,

$$
\sigma_{1}^{\#}\left(x^{1}, x^{2}\right) \leq c 2^{2 N+m+l} .
$$


Suppose $2 N+m+l \leq \lambda$. Then

$$
\sigma_{1}^{\#}\left(x^{1}, x^{2}\right) \leq c 2^{\lambda} \quad \text { and } \quad \mu\left\{\sigma_{1}^{\#}>c 2^{\lambda}\right\}=0 .
$$

Hence, we can suppose that

$$
m+l>\lambda-2 N
$$

We have

$$
\begin{aligned}
& E:=\sum_{n=0}^{N-1} \sum_{m=0}^{n} \mu\left\{\left(x^{1}, x^{2}\right) \in\left(I_{m} \backslash I_{m+1}\right) \times\left(I_{n} \backslash I_{n+1}\right): \sigma_{1}^{\#}\left(x^{1}, x^{2}\right)>c 2^{\lambda}\right\} \\
& \leq c \sum_{n=0}^{N-1} \sum_{m=0}^{n} \sum_{l=m+1, m+l>\lambda-2 N}^{n+1} \sum_{x_{n+1}^{1}=0}^{1} \ldots \sum_{x_{N-1}^{1}=0}^{1} \\
& \mu\left\{\left(x^{1}, x^{2}\right) \in I_{N}\left(\overline{0}, x_{m}^{1}=1, \overline{0}, x_{l}^{1}=1, \overline{0}, x_{n+1}^{1}, \ldots, x_{N-1}^{1}\right)\right. \\
&\left.\times I_{N}\left(\overline{0}, x_{n}^{2}=1, x_{n+1}^{1}, \ldots, x_{N-1}^{1}\right)\right\} .
\end{aligned}
$$

Define

$$
A:=\{(l, m): m+l>\lambda-2 N\}, \quad B:=\{(l, m): 0 \leq l \leq n, 0 \leq m \leq l\} .
$$

Suppose $\lambda-2 N \leq 0$. Then it is evident that

$$
A \cap B=\{(l, m): 0 \leq l \leq n, 0 \leq m \leq l\} .
$$

Hence, we can write

$$
E \leq c \sum_{n=0}^{N-1} \sum_{l=0}^{n} \sum_{m=0}^{l} \frac{2^{N-n}}{2^{2 N}} \leq \frac{c}{2^{N}} \sum_{n=0}^{N-1} \frac{n^{2}}{2^{n}} \leq \frac{c}{2^{N}} \leq \frac{c}{2^{\lambda / 2}} .
$$

Suppose $\lambda-2 N>0$ and $0 \leq n<(\lambda-2 N) / 2$. Then it is easy to show that

$$
A \cap B=\emptyset .
$$

Suppose $\lambda-2 N>0$ and $(\lambda-2 N) / 2 \leq n<\lambda-2 N$. Then we can write

$$
A \cap B=\{(l, m):(\lambda-2 N) / 2 \leq l \leq n, \lambda-2 N-l \leq m \leq l\} .
$$

Consequently,

$$
\begin{aligned}
E & \leq c \sum_{n=[\lambda / 2]-N}^{[\lambda]-2 N} \sum_{l=[\lambda / 2]-N}^{n} \sum_{m=[\lambda]-2 N-l}^{l} \frac{2^{N-n}}{2^{2 N}} \\
& \leq \frac{c}{2^{N}} \sum_{n=[\lambda / 2]-N}^{[\lambda]-2 N} \frac{(n-(\lambda / 2-N))^{2}}{2^{n}} \leq \frac{c}{2^{N} 2^{\lambda / 2-N}} \leq \frac{c}{2^{\lambda / 2}} .
\end{aligned}
$$


Suppose $\lambda-2 N>0$ and $\lambda-2 N \leq n<N$. Then it is evident that

$$
\begin{aligned}
A \cap B= & \{(l, m):(\lambda-2 N) / 2 \leq l \leq \lambda-2 N, \lambda-2 N-l \leq m \leq l\} \\
& \cup\{(l, m): \lambda-2 N<l \leq n, 0 \leq m \leq l\} .
\end{aligned}
$$

Consequently, we can write

$$
\begin{aligned}
E \leq & c \sum_{n=[\lambda]-2 N}^{N-1} \sum_{l=[\lambda / 2]-N}^{[\lambda]-2 N} \sum_{m=[\lambda]-2 N-l}^{l} \frac{2^{N-n}}{2^{2 N}} \\
& +c \sum_{n=[\lambda]-2 N}^{N-1} \sum_{l=[\lambda]-2 N}^{n} \sum_{m=0}^{l} \frac{2^{N-n}}{2^{2 N}} \\
\leq & \frac{c}{2^{N}} \frac{(\lambda / 2-N)^{2}}{2^{\lambda-2 N}} \leq \frac{c}{2^{\lambda / 2}}
\end{aligned}
$$

Combining (3)-(6) we obtain

$$
\begin{aligned}
\sum_{n=0}^{N-1} \sum_{m=0}^{n} \mu\left\{\left(x^{1}, x^{2}\right) \in\left(I_{m} \backslash I_{m+1}\right) \times\left(I_{n} \backslash I_{n+1}\right): \sigma^{\#} a\left(x^{1}, x^{2}\right)\right. & \left.>c 2^{\lambda}\right\} \\
& \leq c / 2^{\lambda / 2}
\end{aligned}
$$

Analogously, we can prove that

$$
\begin{aligned}
& \sum_{n=0}^{N-1} \sum_{m=n}^{N-1} \mu\left\{\left(x^{1}, x^{2}\right) \in\left(I_{m} \backslash I_{m+1}\right) \times\left(I_{n} \backslash I_{n+1}\right): \sigma^{\#} a\left(x^{1}, x^{2}\right)\right.\left.>c 2^{\lambda}\right\} \\
& \leq c / 2^{\lambda / 2}
\end{aligned}
$$

From (7) and (8) we get

$$
\mu\left\{\left(x^{1}, x^{2}\right) \in \bar{I}_{N} \times \bar{I}_{N}:\left|\sigma^{\#} a\left(x^{1}, x^{2}\right)\right|>c 2^{\lambda}\right\} \leq c / 2^{\lambda / 2} .
$$

Let $\left(x^{1}, x^{2}\right) \in I_{N} \times\left(I_{l} \backslash I_{l+1}\right)$. Then from Lemma 7 we have

$$
\begin{aligned}
\sigma^{\#} a\left(x^{1}, x^{2}\right) & \leq c 2^{4 N} \sup _{A>N} \int_{I_{N} \times I_{N}} K_{2^{A}}\left(x^{1}+t^{1}, x^{2}+t^{2}\right) d \mu\left(t^{1}, t^{2}\right) \\
& \leq \frac{c 2^{4 N+l}}{2^{3 N}} \sum_{m=l+1}^{N} 2^{m} D_{2^{N}}\left(x^{2}+e_{l}+e_{m}\right) \\
& =c 2^{N+l} \sum_{m=l+1}^{N} 2^{m} D_{2^{N}}\left(x^{2}+e_{l}+e_{m}\right) .
\end{aligned}
$$

Define

$$
\sigma_{2}^{\#}\left(x^{1}, x^{2}\right):=c 2^{N+l} \sum_{m=l+1}^{N} 2^{m} D_{2^{N}}\left(x^{2}+e_{l}+e_{m}\right) .
$$


From (1) we can write

$$
D_{2^{N}}\left(x^{2}+e_{l}+e_{m}\right)= \begin{cases}2^{n}, & x^{2} \in I_{N}\left(\overline{0}, x_{l}=1, \overline{0}, x_{m}=1, \overline{0}\right), \\ 0, & x^{2} \notin I_{N}\left(\overline{0}, x_{l}=1, \overline{0}, x_{m}=1, \overline{0}\right) .\end{cases}
$$

Therefore

$$
\sigma_{2}^{\#}\left(x^{1}, x^{2}\right) \neq 0
$$

implies that

$$
x^{2} \in I_{N}\left(\overline{0}, x_{l}=1, \overline{0}, x_{m}=1, \overline{0}\right)
$$

for some $m$ with $l<m \leq N$. Consequently,

$$
\sigma_{2}^{\#}\left(x^{1}, x^{2}\right) \leq c 2^{l+2 N+m} .
$$

Suppose $l+2 N+m \leq \lambda$. Then

$$
\sigma_{2}^{\#}\left(x^{1}, x^{2}\right) \leq c 2^{\lambda} \quad \text { and } \quad \mu\left\{\sigma_{2}^{\#}>c 2^{\lambda}\right\}=0 .
$$

Hence, we can suppose that

$$
l+2 N+m>\lambda
$$

Define

$$
T:=\{(m, l): l+m>\lambda-2 N\}, \quad S:=\{(m, l): 0 \leq l \leq m<N\} .
$$

Suppose $\lambda-2 N \leq 0$. Then it is evident that

$$
T \cap S=\{(m, l): 0 \leq m<N, 0 \leq l \leq m\} .
$$

Hence

$$
\begin{aligned}
& \sum_{l=0}^{N-1} \mu\left\{\left(x^{1}, x^{2}\right) \in I_{N} \times\left(I_{l} \backslash I_{l+1}\right): \sigma^{\#} a\left(x^{1}, x^{2}\right)>c 2^{\lambda}\right\} \\
& \quad \leq \sum_{l=0}^{N-1} \sum_{m=l+1}^{N-1} \mu\left\{\left(x^{1}, x^{2}\right) \in I_{N} \times I_{N}\left(\overline{0}, x_{l}=1, \overline{0}, x_{m}=1, \overline{0}\right):\right. \\
& \quad \leq c \sum_{l=0}^{N-1} \sum_{m=l+1}^{N-1} \frac{1}{2^{2 N}} \leq \frac{c N^{2}}{2^{2 N}}<\frac{c}{2^{N}}<\frac{c}{2^{\lambda / 2}} .
\end{aligned}
$$

Suppose $2 N<\lambda \leq 3 N$. Then it is easy to show that

$$
\begin{aligned}
T \cap S= & \{(m, l): \lambda / 2-N \leq m<\lambda-2 N, \lambda-2 N-m \leq l \leq m\} \\
& \cup\{(m, l): \lambda-2 N \leq m<N, 0 \leq l \leq m\} .
\end{aligned}
$$


Consequently,

$$
\sum_{l=0}^{N-1} \mu\left\{\left(x^{1}, x^{2}\right) \in I_{N} \times\left(I_{l} \backslash I_{l+1}\right): \sigma^{\#} a\left(x^{1}, x^{2}\right)>c 2^{\lambda}\right\}
$$

$\leq \sum_{m=[\lambda / 2]-N}^{[\lambda]-2 N} \sum_{l=[\lambda]-2 N-m}^{m} \mu\left\{\left(x^{1}, x^{2}\right) \in I_{N} \times I_{N}\left(\overline{0}, x_{l}=1, \overline{0}, x_{m}=1, \overline{0}\right):\right.$
$\left.\sigma^{\#} a\left(x^{1}, x^{2}\right)>c 2^{\lambda}\right\}$

$+\sum_{m=[\lambda]-2 N}^{N-1} \sum_{l=0}^{m} \mu\left\{\left(x^{1}, x^{2}\right) \in I_{N} \times I_{N}\left(\overline{0}, x_{l}=1, \overline{0}, x_{m}=1, \overline{0}\right):\right.$ $\left.\sigma^{\#} a\left(x^{1}, x^{2}\right)>c 2^{\lambda}\right\}$

$\leq c \sum_{m=[\lambda / 2]-N}^{[\lambda]-2 N} \sum_{l=[\lambda]-2 N-m}^{m} \frac{1}{2^{2 N}}+\sum_{m=[\lambda]-2 N}^{N-1} \sum_{l=0}^{m} \frac{1}{2^{2 N}}$

$\leq c \sum_{m=[\lambda / 2]-N}^{[\lambda]-2 N} \frac{m-(\lambda / 2-N)}{2^{2 N}}+\frac{c N^{2}}{2^{2 N}} \leq \frac{c N^{2}}{2^{2 N}} \leq \frac{c \lambda^{2}}{2^{(2 / 3) \lambda}} \leq \frac{c}{2^{\lambda / 2}}$.

Suppose $\lambda>3 N$. Then

$$
T \cap S=\{(m, l): \lambda / 2-N \leq m<N, \lambda-2 N-m \leq l \leq m\} .
$$

Consequently,

$$
\begin{aligned}
& \sum_{l=0}^{N-1} \mu\left\{\left(x^{1}, x^{2}\right) \in I_{N} \times\left(I_{l} \backslash I_{l+1}\right): \sigma^{\#} a\left(x^{1}, x^{2}\right)>c 2^{\lambda}\right\} \\
& \quad \leq c \sum_{m=[\lambda / 2]-N}^{N-1} \sum_{l=[\lambda]-2 N-m}^{m} \frac{1}{2^{2 N}} \leq \frac{c(2 N-\lambda / 2)^{2}}{2^{2 N-\lambda / 2}} \frac{1}{2^{\lambda / 2}} \leq \frac{c}{2^{\lambda / 2}} .
\end{aligned}
$$

Combining (11)-(13) we obtain

$$
\mu\left\{\left(x^{1}, x^{2}\right) \in I_{N} \times \bar{I}_{N}:\left|\sigma^{\#} a\left(x^{1}, x^{2}\right)\right|>c 2^{\lambda}\right\} \leq c / 2^{\lambda / 2} .
$$

Analogously, we can prove that

$$
\mu\left\{\left(x^{1}, x^{2}\right) \in \bar{I}_{N} \times I_{N}:\left|\sigma^{\#} a\left(x^{1}, x^{2}\right)\right|>c 2^{\lambda}\right\} \leq c / 2^{\lambda / 2} .
$$

From (9), (14) and (15) we obtain

$$
\mu\left\{\left(x^{1}, x^{2}\right) \in \overline{I_{N} \times I_{N}}:\left|\sigma^{\#} a\left(x^{1}, x^{2}\right)\right|>c 2^{\lambda}\right\} \leq c / 2^{\lambda / 2} .
$$

Theorem 1 is proved.

Proof of Theorem 2. Let $A \in \mathbb{P}$ and

$$
f_{A}\left(x^{1}, x^{2}\right):=\left(D_{2^{A+1}}\left(x^{1}\right)-D_{2^{A}}\left(x^{1}\right)\right)\left(D_{2^{A+1}}\left(x^{2}\right)-D_{2^{A}}\left(x^{2}\right)\right) .
$$


It is evident that

$$
\widehat{f}_{A}(i, k)= \begin{cases}1 & \text { if } i, k=2^{A}, \ldots, 2^{A+1}-1, \\ 0 & \text { otherwise }\end{cases}
$$

Then we can write that

(16) $S_{k, k}\left(f_{A} ; x^{1}, x^{2}\right)$

$$
\begin{cases}0 & \text { if } k=0, \ldots, 2^{A}, \\ \left(D_{k}\left(x^{1}\right)-D_{2^{A}}\left(x^{1}\right)\right)\left(D_{k}\left(x^{2}\right)-D_{2^{A}}\left(x^{2}\right)\right) & \text { if } k=2^{A}+1, \ldots, 2^{A+1}-1, \\ f_{A}\left(x^{1}, x^{2}\right) & \text { if } k \geq 2^{A+1}\end{cases}
$$

We have

$$
\begin{gathered}
f_{A}^{*}\left(x^{1}, x^{2}\right)=\sup _{k}\left|S_{2^{k}, 2^{k}}\left(f_{A} ; x^{1}, x^{2}\right)\right|=\left|f_{A}\left(x^{1}, x^{2}\right)\right|, \\
\left\|f_{A}\right\|_{H_{p}}=\left\|f_{A}^{*}\right\|_{p}=\left\|D_{2^{A}}\right\|_{p}^{2}=2^{2 A(1-1 / p)} .
\end{gathered}
$$

Since

$$
D_{k+2^{A}}-D_{2^{A}}=w_{2^{A}} D_{k}, \quad k=1, \ldots, 2^{A},
$$

from (16) we obtain

$$
\begin{aligned}
\sigma^{\#} f_{A}\left(x^{1}, x^{2}\right) & =\sup _{n}\left|\sigma_{2^{n}}\left(f_{A} ; x^{1}, x^{2}\right)\right| \geq \sigma_{2^{A+1}}\left(f_{A} ; x^{1}, x^{2}\right) \\
& =\frac{1}{2^{A+1}}\left|\sum_{k=0}^{2^{A+1}-1} S_{k, k}\left(f_{A} ; x^{1}, x^{2}\right)\right| \\
& =\frac{1}{2^{A+1}}\left|\sum_{k=2^{A}+1}^{2^{A+1}-1}\left(D_{k}\left(x^{1}\right)-D_{2^{A}}\left(x^{1}\right)\right)\left(D_{k}\left(x^{2}\right)-D_{2^{A}}\left(x^{2}\right)\right)\right| \\
& =\frac{1}{2^{A+1}}\left|\sum_{k=1}^{2^{A}-1}\left(D_{k+2^{A}}\left(x^{1}\right)-D_{2^{A}}\left(x^{1}\right)\right)\left(D_{k+2^{A}}\left(x^{2}\right)-D_{2^{A}}\left(x^{2}\right)\right)\right| \\
& =\frac{1}{2^{A+1}}\left|\sum_{k=1}^{2^{A}-1} D_{k}\left(x^{1}\right) D_{k}\left(x^{2}\right)\right|=\frac{1}{2}\left|K_{2^{A}}\left(x^{1}, x^{2}\right)\right| .
\end{aligned}
$$

Let

$$
\begin{aligned}
\left(x^{1}, x^{2}\right) \in & I_{A}\left(\overline{0}, x_{m}^{1}=1, \overline{0}, x_{n}^{1}=1, x_{n+1}^{1}, \ldots, x_{A-1}^{1}\right) \\
& \times I_{A}\left(\overline{0}, x_{n}^{2}=1, x_{n+1}^{1}, \ldots, x_{A-1}^{1}\right) .
\end{aligned}
$$

Then from Lemma 2 we obtain

$$
\left|K_{2^{A}}\left(x^{1}, x^{2}\right)\right|=2^{m+n-2} .
$$


Hence we can write

$$
\begin{aligned}
& \int_{G \times G}\left|K_{2^{A}}\left(x^{1}, x^{2}\right)\right|^{1 / 2} d \mu\left(x^{1}, x^{2}\right) \\
& \geq \sum_{m=0}^{A-1} \sum_{n=m+1}^{A-1} \sum_{x_{n+1}^{1}=0}^{1} \cdots \sum_{x_{A-1}^{1}=0}^{1} \int_{I_{A}\left(\overline{0}, x_{m}^{1}=1, \overline{0}, x_{n}^{1}=1, x_{n+1}^{1}, \ldots, x_{A-1}^{1}\right) \times I_{A}\left(\overline{0}, x_{n}^{1}=1, x_{n+1}^{1}, \ldots, x_{A-1}^{1}\right)}\left|K_{2^{A}}\left(x^{1}, x^{2}\right)\right|^{1 / 2} d \mu\left(x^{1}, x^{2}\right) \\
& =\sum_{m=0}^{A-1} \sum_{n=m+1}^{A-1} 2^{(m+n-2) / 2} \sum_{x_{n+1}^{1}=0}^{1} \ldots \sum_{x_{A-1}^{1}=0}^{1} \int_{G \times G} 1_{I_{A}\left(\overline{0}, x_{m}^{1}=1, \overline{0}, x_{n}^{1}=1, x_{n+1}^{1}, \ldots, x_{A-1}^{1}\right)}\left(x^{1}\right) \\
& \geq c \sum_{m=0}^{A-1} 2_{I_{A}\left(\overline{0}, x_{n}^{2}=1, x_{n+1}^{1}, \ldots, x_{A-1}^{1}\right)}\left(x^{2}\right) d \mu\left(x^{1}, x^{2}\right) \\
& \sum_{n=m}^{A-1} 2^{n / 2} \frac{1}{2^{2 A}} 2^{A-n} \geq \frac{c A}{2^{A}},
\end{aligned}
$$

and

$$
\frac{\left\|\sigma^{\#} f_{A}\right\|_{1 / 2}}{\left\|f_{A}\right\|_{1 / 2}} \geq \frac{c A^{2}}{2^{2 A} 2^{2 A(1-2)}} \geq c A^{2} \rightarrow \infty \quad \text { as } A \rightarrow \infty .
$$

Theorem 2 is proved.

Since $\sigma^{\#}$ is bounded from $L_{\infty}(G \times G)$ to $L_{\infty}(G \times G)$ the validity of Corollaries 3 and 4 follows by interpolation (see Weisz [13]) from Theorems 1 and 2.

Acknowledgments. The author wishes to express his gratitude to the referees for helpful remarks.

\section{References}

[1] J. Fine, Cesàro summability of Walsh-Fourier series, Proc. Nat. Acad. Sci. USA 41 (1955), 558-591.

[2] N. J. Fujii, Cesàro summability of Walsh-Fourier series, Proc. Amer. Math. Soc. 77 (1979), 111-116.

[3] G. Gát, Convergence of Marcinkiewicz means of integrable functions with respect to two-dimensional Vilenkin systems, Georgian Math. J. 11 (2004), 467-478.

[4] U. Goginava, Marcinkiewicz-Fejér means of d-dimensional Walsh-Fourier series, J. Math. Anal. Appl. 307 (2005), 206-218.

[5] K. Nagy, Some convergence properties of the Walsh-Kaczmarz system with respect to the Marcinkiewicz means, Rend. Circ. Mat. Palermo (2) Suppl. 76 (2005), 503-516.

[6] F. Schipp, Certain rearrangements of series in the Walsh system, Mat. Zametki 18 (1975), 193-201 (in Russian).

[7] F. Schipp, W. R. Wade, P. Simon and J. Pál, Walsh Series, an Introduction to Dyadic Harmonic Analysis, Adam Hilger, Bristol, 1990.

[8] P. Simon, Investigations with respect to the Vilenkin system, Ann. Univ. Sci. Budapest. Eötvös Sect. Math. 27 (1984), 87-101. 
[9] P. Simon, Cesàro summability with respect to two-parameter Walsh system, Monatsh. Math. 131 (2000), 321-334.

[10] F. Weisz, Martingale Hardy Spaces and their Applications in Fourier Analysis, Springer, Berlin, 1994.

[11] —, Cesàro summability of one and two-dimensional Walsh-Fourier series, Anal. Math. 22 (1996), 229-242.

[12] - Convergence of double Walsh-Fourier series and Hardy spaces, Approx. Theory Appl. 17 (2001), no. 2, 32-44.

[13] - Summability of Multi-Dimensional Fourier Series and Hardy Spaces, Kluwer, Dordrecht, 2002.

[14] —, $\vartheta$-summability of Fourier series, Acta Math. Hungar. 103 (2004), 139-176.

Department of Mechanics and Mathematics

Tbilisi State University

Chavchavadze St. 1

Tbilisi 0128, Georgia

E-mail: z_goginava@hotmail.com

Received November 25, 2005

Revised version January 20, 2008 